\title{
Carnets
}

Revue électronique d'études françaises de l'APEF

Deuxième série - 15 | 2019

Jules Verne et les pouvoirs de l'imagination

\section{Imagination et bande dessinée}

Vingt mille lieues sous les mers

\section{María-Pílar Tresaco et Ana-Isabel Moniz}

\section{(2) OpenEdition}

Journals

Édition électronique

URL : http://journals.openedition.org/carnets/9415

DOI : $10.4000 /$ carnets. 9415

ISSN : 1646-7698

Éditeur

APEF

Référence électronique

María-Pílar Tresaco et Ana-Isabel Moniz, «Imagination et bande dessinée », Carnets [En ligne], Deuxième série - 15 | 2019, mis en ligne le 31 janvier 2019, consulté le 20 avril 2019. URL : http:// journals.openedition.org/carnets/9415; DOI : 10.4000/carnets.9415

Ce document a été généré automatiquement le 20 avril 2019

\section{(c) (†) (8)}

Carnets est mis à disposition selon les termes de la licence Creative Commons - Atribution - Pas d'utilisation commerciale 4.0 International. 


\title{
Imagination et bande dessinée
}

\author{
Vingt mille lieues sous les mers \\ María-Pílar Tresaco et Ana-Isabel Moniz
}

\section{NOTE DE L'AUTEUR}

T3AxEL, groupe de recherches de l'Université de Saragosse. Financement : Gouvernement d'Aragon /FEDER.

1 L'imagination, considérée par Baudelaire comme «la reine des facultés » (1976:620), permet de construire un univers fictionnel où nos propres idées jouent un rôle déterminant. C'est ce qui arrive lorsqu'un adaptateur et un illustrateur modifient une œuvre comme Vingt mille lieues sous les mers à travers le processus d'illustration du texte, où la cohérence intersémiotique joue un rôle déterminant dans le dialogue établi entre les signifiés dénotatifs et connotatifs.

Trois éléments participent de manière systématique dans la communication écrite : le texte, l'image et le champ visuel où ils s'unissent. Pour transmettre un message, nous avons besoin de codifier l'information et cette opération se fait de manière essentiellement visuelle lorsqu'il s'agit d'œuvres de bandes dessinées. En tant que séquence d'images fixes, la bande dessinée se présente comme une forme de narration figurative dans laquelle l'image s'impose - ou semble s'imposer - à l'écrit. L'image devient-elle écriture et signes tout comme « Le monde du dessein n'est plus alors que le décor dans lequel s'incarnent les paroles » (Fresnault-Deruelle, 1970 : 154).

2 L'information du texte littéraire a des buts différents et ne peut pas correspondre entièrement à celle d'une adaptation. De plus, un roman présente des situations complexes qui n'apparaissent pas entièrement versées dans une adaptation. En effet, la symbiose entre les auteurs est difficilement atteinte et la finalité de leur discours ne coïncide pas. Il est vrai qu'il y a un énorme travail esthétique dérivé de l'imagination et de l'optique créative de l'adaptateur et de l'illustrateur. Cependant, cette activité doit reproduire l'idée que l'écrivain a décrite dans son roman. Or, nous ne cherchons pas seulement à savoir ce que l'auteur veut nous dire, nous devons aussi comprendre ce que 
l'adaptation transmet et si elle décrit ce que le roman original raconte. Vu ainsi, le texte qui accompagne l'image aide le lecteur à trouver un sens - une lecture possible - parmi les plusieurs possibilités que celle-ci offre au lecteur :

le texte [qui accompagne les images] dirige le lecteur entre les signifiés de l'image, lui en fait éviter certains et en recevoir d'autres; à travers un dispatching souvent subtil, il le téléguide vers un sens choisi à l'avance. Dans tous ces cas d'ancrage, le langage a évidemment une fonction d'élucidation, mais cette élucidation est sélective ; il s'agit d'un meta-langage appliqué non à la totalité du message iconique, mais seulement à certains de ses signes (Barthes, $1964: 44$ ).

Nous essayerons donc de voir la fonction du langage écrit et de celle de l'illustration dans l'œuvre de Jules Verne. Les traductions et adaptations de Vingt mille lieues sous les mers, roman publié en France entre 1869 et 1870, sont très nombreuses ainsi que les bandes dessinées adaptées de ce roman. C'est pourquoi nous nous focaliserons simplement sur les premières de couverture que nous nommerons dorénavant couvertures.

En tant que synthèse de l'adaptation proposée par l'illustrateur à l'intérieur du livre, la couverture appelle aussitôt à notre imagination et nous permet de construire l'histoire que nous allons découvrir par la suite. L'information transmise par la couverture aura une source et une destination différentes dans le temps et dans l'espace. Il faut donc tenir en ligne de compte le lecteur de chaque époque. Ces informations relèvent d'un code spécifique (L.F. Cao, 1998: 40), celui produit par un émetteur - ici l'adaptateur et/ou l'illustrateur -, et le récepteur, le lecteur en l'occurrence, transformera cette information en la décodant. Avant de commenter ces éditions de Vingt mille lieues sous les mers, nous allons observer la première adaptation en portugais, publiée en 1946, sous la forme de feuilleton dans Diabrete (fig. 1).

Cette couverture réunit les quatre personnages principaux du roman. Le poulpe, qui tient en l'air un marin, sépare d'un côté Nemo (à gauche) et de l'autre Ned Land, Conseil et Aronnax (à droite, de haut en bas). Le tout est entouré de trois mots - sensacional, formidavel, maravilhoso - Ce trois mots clés captent l'attention du lecteur. Ce sont trois signes essentiels qui nous permettent d'entrer aussitôt dans histoire que l'auteur va raconter.

Diabrete, 1946 (fig. 1)

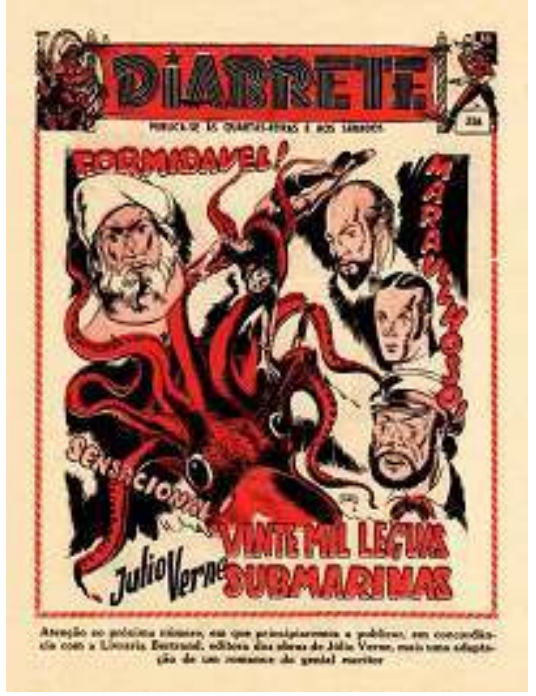


4 L'image centrale représente la férocité du poupe face au dramatisme de la mort. Sa couverture, noire et rouge, nous annonce la façon dont les couvertures suivantes vont se présenter. L'utilisation de la couleur implique également un fort pourcentage de subjectivité car chaque lecteur réagit de manière différente à un même stimulus chromatique. L'emploi de la couleur se revêt ainsi d'une énorme importance, de par aussi son impact visuel.

Pour expliquer cette idée, nous avons choisi la première adaptation espagnole en bande dessinée. Publiée en $1955_{\mathrm{a}}$ par Valor ediciones, sous la forme de feuilleton, en huit fascicules en couleur, elle s'annonçait comme «Aventuras Fantásticas» (Aventures Fantastiques). Chaque fascicule a quinze pages et toutes les deux pages il y a une alternance de couleur : une page est en couleur et l'autre noir et blanc L'ensemble des fascicules a, de son côté, une première de couverture (fig. 2).

Valor Ediciones, 1955 (fig. 2)

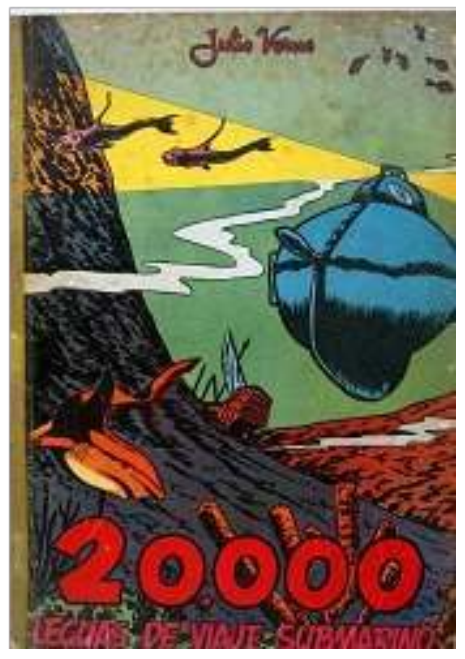

5 L'illustrateur des quatre premiers fascicules est Francisco Javier. Toutefois, les quatre suivants ne sont pas signés. Si nous observons les quatre premières couvertures (fig. 3),

Fascicules 1, 2, 3 et 4 (fig. 3)

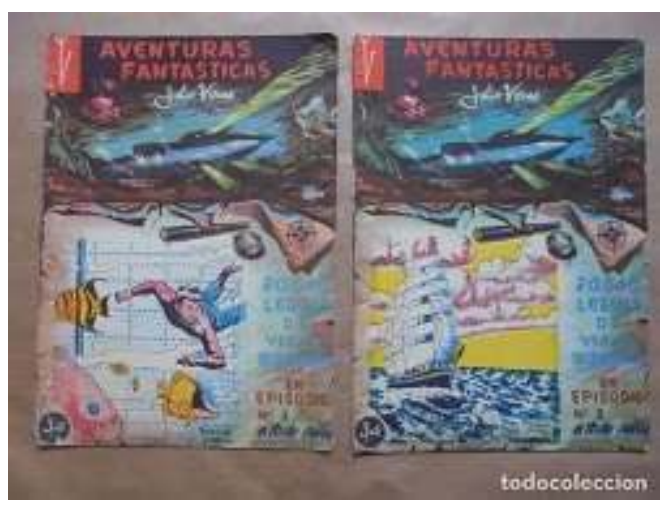




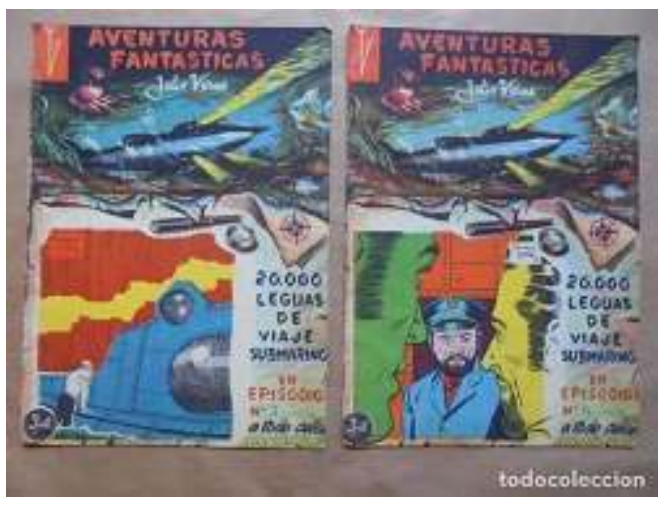

nous remarquons que la distribution des images occupe sensiblement le même espace que le texte écrit. Le rouge attire notre attention, mais il est utilisé essentiellement pour le titre générique face au bleu qui est employé pour le titre du roman. Nous y avons, selon Abraham Moles (Aparici \& García, 1998 : 90), la violence et l'enthousiasme du rouge face à la froide sérénité du bleu.

Les quatre couvertures suivantes (fig.4) ont une représentation graphique bien différente.

Fascicules 5, 6, 7 et 8 (fig. 4)
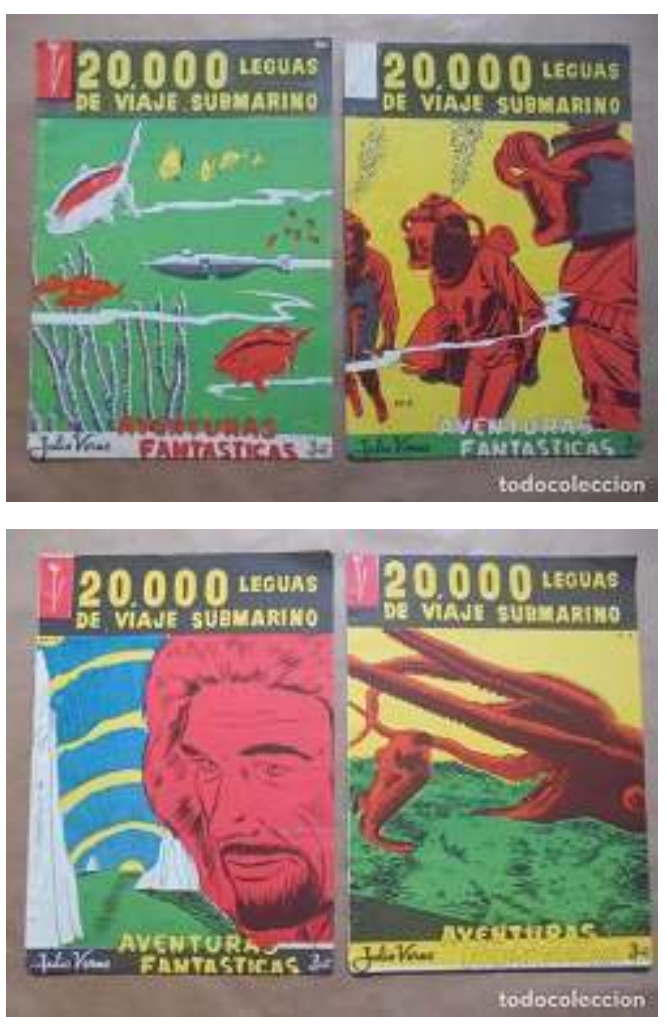

Maintenant c'est le titre du roman, en lettres capitales, qui prend de l'importance dans la couverture. De plus, on utilise le jaune, couleur stimulante et lumineuse, pour attirer l'attention du lecteur.

La couleur rouge des couvertures des fascicules 6,7 et 8 fait ressortir la valeur des trois éléments distinctifs du roman : les plongeurs, le capitaine Nemo et le poulpe. Cependant, ce qui attire l'attention de l'image 5 est la présence du Nautilus. Il occupe une position 
centrale et le sous-marin est entouré d'images rouges. Le graphisme de cette présentation s'adresse essentiellement à l'affectivité du récepteur. En utilisant ainsi les couleurs et cette disposition on capte d'emblée l'attention sur de ce que l'on veut nous montrer.

Si nous comparons chacune des huit couvertures avec la narration correspondante, nous constatons qu'il y a un parallélisme temporel. Par exemple, les glaces dans l'image 7 nous annonce les problèmes de Nautilus. Le rouge de la face de Nemo de la couverture exprime la colère ainsi que le courage qu'il doit avoir pour continuer sa traversée sous-marine. Du côté gauche, les couleurs prédominantes sont le bleu, le jaune et l'échelle des verts. La répétition persistante d'éléments à l'horizon conduit à une composition harmonieuse, capable de produire un effet sensoriel sémantique, de façon à pouvoir parler d'une allitération visuelle.

Stéphanie Clément (2013 : note 6, p.62) affirme que

Quand les calmars attaquent le Nautilus les images utilisées pour décrire l'accident jouent de l'amalgame entre le récit des évènements et les évènements eux-mêmes : la victime est brandie " comme une plume ", l'animal lance " un liquide noirâtre " et, enfin, se répandent sur le sol des flots « d'encre noire » (Verne, $1871: 396)$.

Alphonse de Neuville est l'illustrateur de l'édition d'Hetzel (fig. 5).

Hetzel, 1871 (fig. 5)
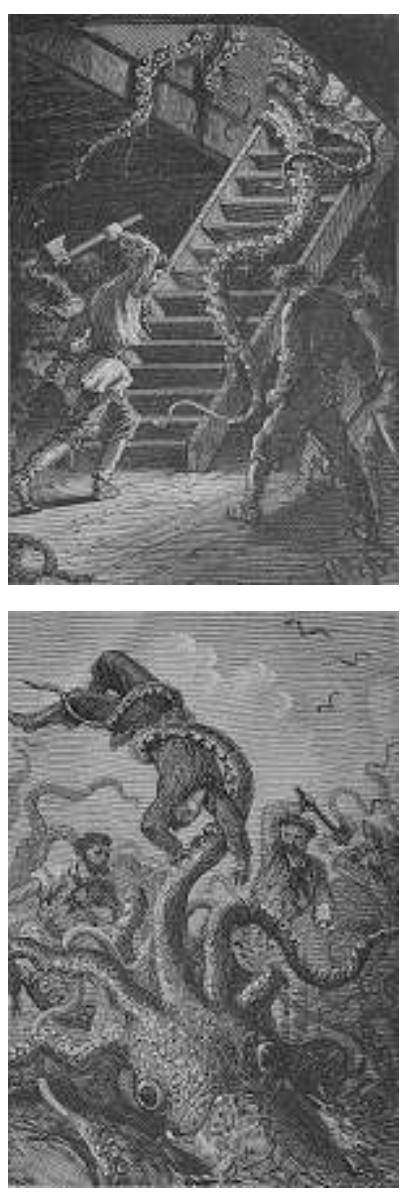

9 La représentation de cette description va se répéter sur de nombreuses couvertures mais avec des variantes très visibles.

Les couvertures de l'adaptation en bande dessinée de la collection « Historias » de $1955_{\mathrm{b}}$ (fig. 6) et de la jaquette de la «Colección Historias selección » de 1967 (fig. 7), toutes les 
deux de la maison d'édition Bruguera de Barcelone, montrent le désespoir du marin, car c'est le poulpe qui l'emporte sur l'homme comme chez Hetzel. Ferrán Esteve en est l'illustrateur.

Bruguera, 1955 (fig. 6)

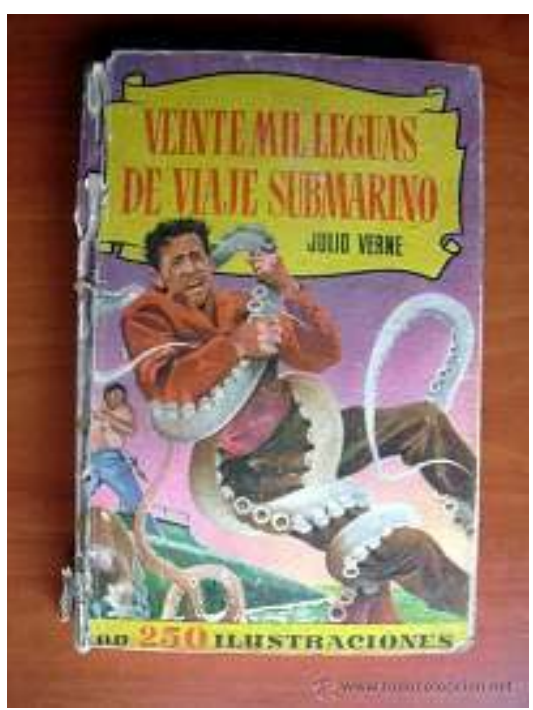

Jaquette de Bruguera, 1967 (fig. 7)

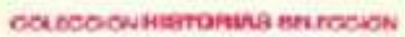

\section{VEINTE MIL}

\section{LEGUAS DE}

VIAJE SUBMARINO

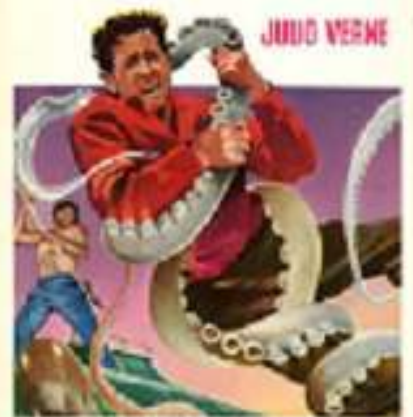

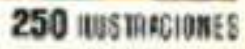

Bruguera réédite cette adaptation de 1955 plusieurs fois. Cependant, le texte et les illustrations intérieures sont toujours les mêmes et la seule variante est l'image de la couverture utilisée dans chaque collection. Cette adaptation a une page sur deux de vignettes qui racontent en parallèle l'histoire du roman.

C'est Tomás Porto qui dessine la couverture de la « Colección Historias selección » de 1972 (fig. 8). Toutefois, il va continuer à transmettre la même idée de son prédécesseur. 


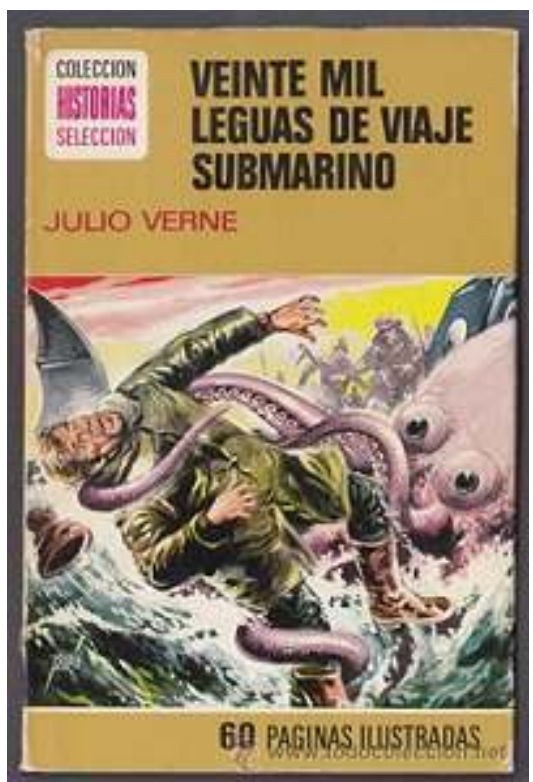

11 Dans l'adaptation de 1983 (fig. 9) de la collection « Historias infantiles », de l'illustrateur Antonio Bernal, c'est tout de même l'homme qui est le vainqueur. Le jaune, qui entoure l'illustration, provoque une réponse active et positive chez le lecteur. Les tonalités verdâtres sont associées à la vigueur; par contre celle du titre, en noir, exprime le désespoir voire même la mort.

Bruguera, 1983 (fig. 9)

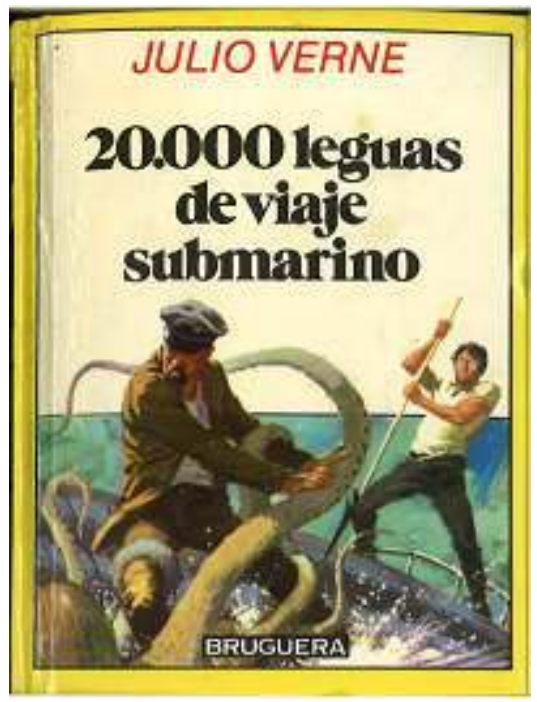

Toutes ces couvertures de Bruguera ont le même sujet. Elles différent donc de l'éditiontexte de ce roman de 1959 , avec douze illustrations à page entière de T. Marco et V. Rosso, et de celle que l'on trouve dans la « colección Iris ». Sur la première de couverture ainsi que sur la jaquette (fig. 10), le lecteur pourra y trouver un motif tout à fait différent : Aronnax observe Nemo jouer de l'organe. Il s'agit d'une image qui ne se répétera plus. Toutefois, sur la page de titre (fig. 11) nous trouvons une scène qui va se reproduire sur bien d'autres couvertures comme nous l'expliquerons ci-dessous. 
Bruguera 1959 (fig. 10)

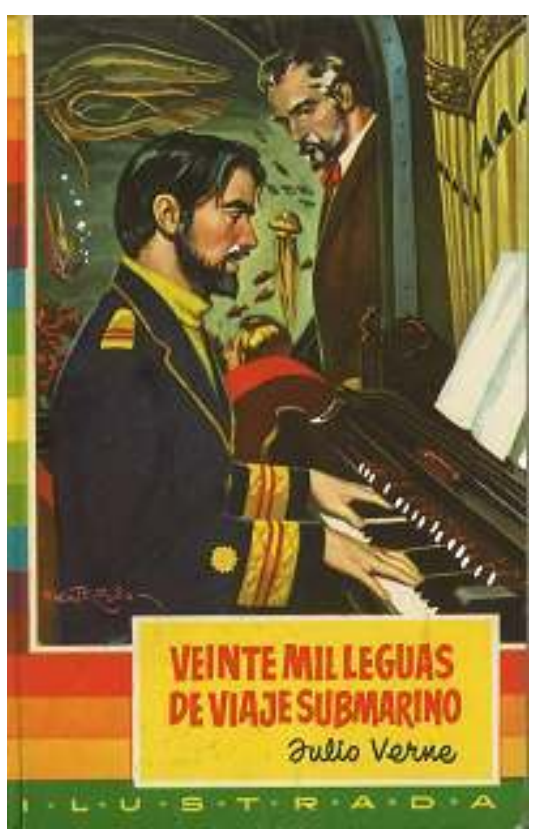

Bruguera 1959a (fig. 11)

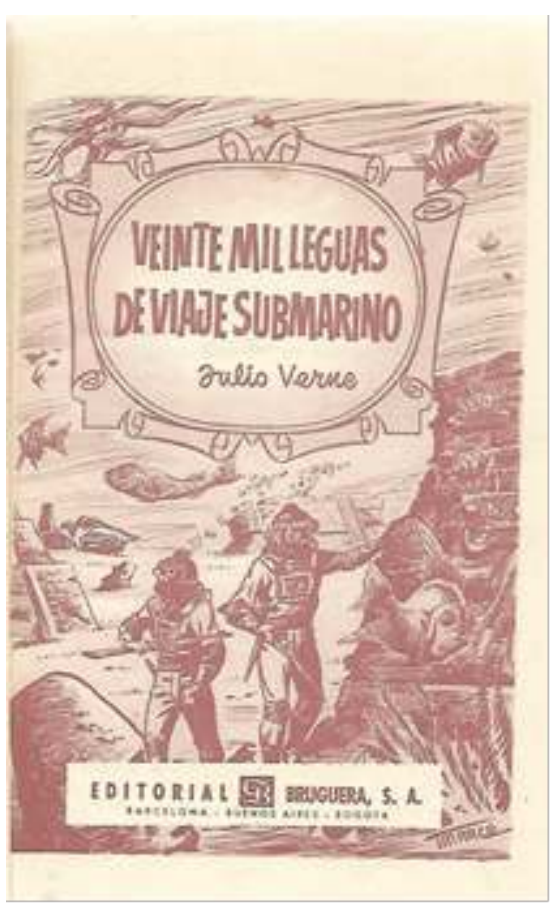

13 D'après les couvertures que nous avons décrites, et si on ne connaît pas l'histoire du roman, on croirait y voir une aventure dont les protagonistes sont les poulpes. D'ailleurs, cette idée se confirme avec la couverture de 1976 de la maison d'édition Laida / Fher de « colección Ediclas J. Verne » en Espagne ; traduite en portugais depuis 1982 chez Edinter (fig. 12), les illustrations appartiennent à Julio Bosch.

Ce grand poulpe joue le rôle principal puisqu'il y il occupe la moitié de l'espace. Nemo et Nautilus ne seront donc pas représentés. Face aux couvertures précédentes, celle-ci n'est 
pas agressive. On peut s'imaginer qu'on va découvrir une aventure d'exploration: une chasse aux trésors.
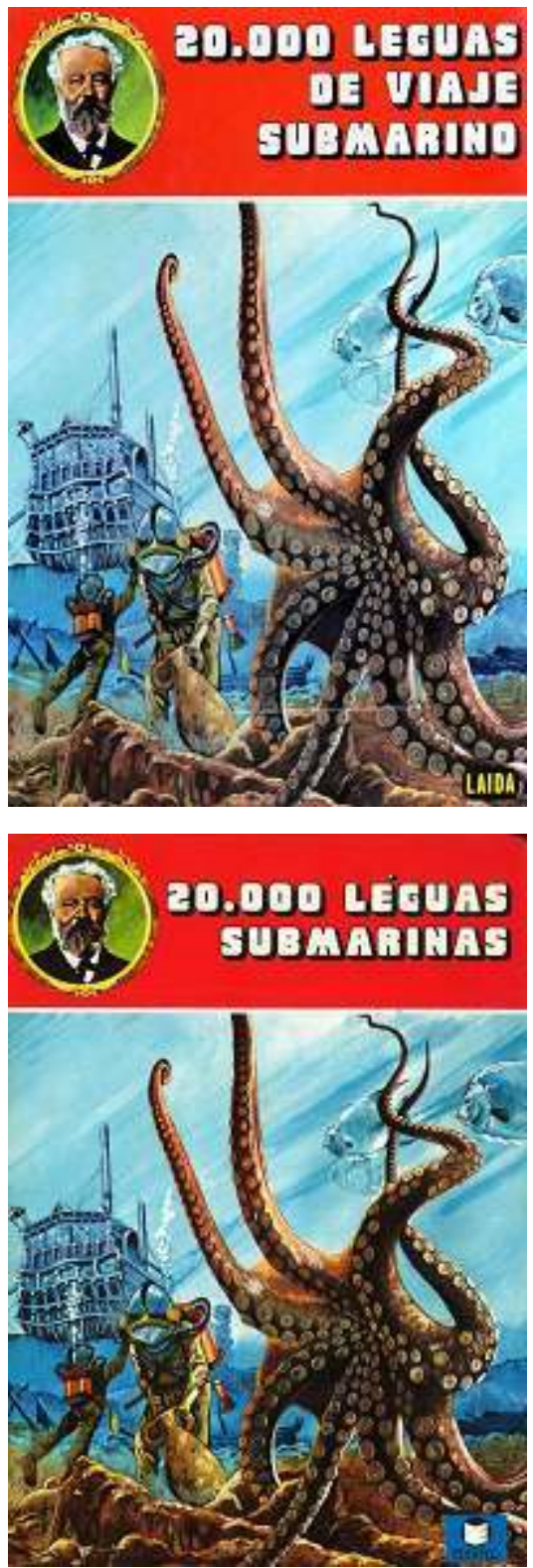

Chaque image a besoin d'une conscience déterminée pour comprendre ce qu'elle représente. Nous avons déjà vu que la rhétorique visuelle nous facilite l'observation d'une allitération, c'est-à-dire la répétition d'un même motif. Cette expression emphatique de la multiplicité d'une idée est présente sur la couverture de Susaeta de 1971, texte et illustrations de Coiras (fig. 13). Nous pouvons y observer une accumulation d'images destinée à frapper le lecteur et à mettre en valeur le pouvoir du poulpe. Il a attrapé quatre plongeurs et l'image nous montre leur impuissance à agir car le poulpe finit par gagner la bataille. Le Nautilus, ainsi que le lecteur, sont de simples observateurs de l'attaque. La position parallèle renforce cette idée. Aussi on peut croire que le Nautilus va disparaître derrière les rochers alors que le poulpe ne va pas lâcher ses victimes. Avec ces couvertures, il nous semble que l'illustrateur et l'adaptateur stimulent l'imagination du 
lecteur avec le combat entre les plongeurs et le poulpe, mais on peut aussi considérer qu'il s'agit d'un subterfuge de marketing.

Susaeta, 1971 (fig. 13)

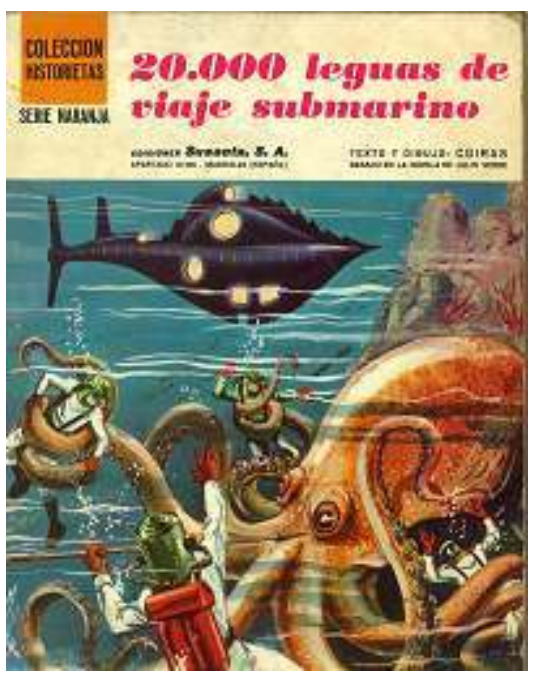

Bien que ce roman de Jules Verne comprenne quarante-sept chapitres seul le XvIII de la deuxième partie - Les poulpes - est consacré à la scène de la lutte avec le poulpe géant ; et pourtant les illustrateurs des adaptations l'ont bien choisi pour le représenter. Une seule de ces adaptations nous raconte le but principal de ce chapitre, à savoir nous révéler qu'il $\mathrm{y}$ avait des français à bord.

Rober O'Connor (2009: 131) trouve que « le génie de Nemo est cloîtré dans ce navire qui manifeste son pouvoir. De même ses découvertes sont destinées à ne pas survivre à l'instrument qui les rend possibles. Son merveilleux vaisseau permet de connaître assez brièvement cette force de spectre ». Or, cette union Nemo - Nautilus n'apparaît jamais sur ces couvertures. Vingt mille lieues sous les mers est tout autant le roman de l'océan que celui du sous-marin. Malgré cela, Nautilus n'occupe aucune place importante sur les couvertures.

Si jusqu' ici, sur toutes les couvertures, le poulpe a déplacé les principaux personnages du roman, maintenant c'est le tour des plongeurs. Daniel Compère (2005: 291) considère que " le scaphandre transforme véritablement l'être humain en nouvel habitant du monde sous-marin ". C'est cette 'idée que les illustrateurs des éditions suivantes vont décrire Publiée par l'Editorial valenciana en 1959 b (fig. 14), l'adaptation de Carlos de Monterroble, avec des illustrations de Brocal Remohi, a une première couverture où aux plongeurs l'illustrateur ajoute une nouvelle image : celle du requin. Mais c'est un requin blessé. Il ne semble donc plus agressif car il a été vaincu, même s'il continue à être un obstacle dangereux pour les plongeurs du Nautilus. 
Editorial valenciana, $1959_{b}$ (fig. 14)

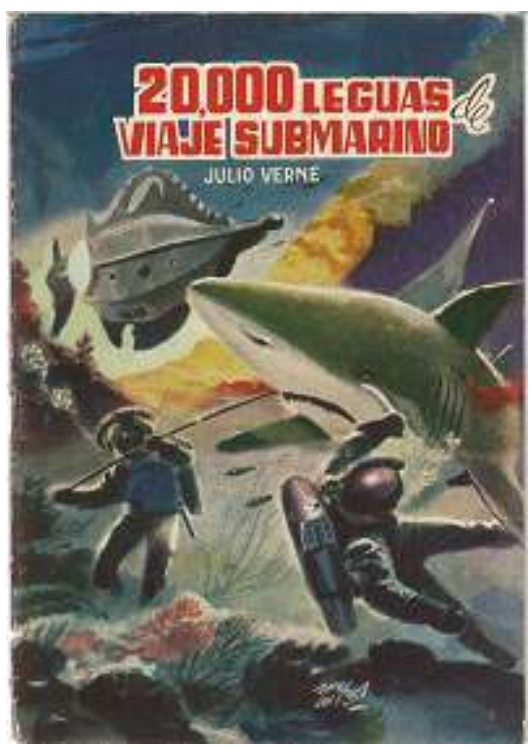

Le sous-marin se trouve derrière une barrière orange, couleur qui, selon Faver Bivien (Aparici \& García, 1998 : 91), exprime le confort et la sécurité. Cette couverture introduit, donc, trois éléments importants, essentiels, du monde sous-marin de Vingt mille lieues sous les mers.

Il s'agit d'une couverture avec sensiblement les mêmes éléments, mais elle apparaît dans un contexte bien plus aimable est celle de Bruguera de 1977 (fig. 15). Il s'agit d'un ensemble de douze romans de Jules Verne, mais la maison d'édition a choisi Vingt mille lieues sous les mers comme présentation de ce volume. L'illustration de cette couverture est aussi d'Antonio Bernal. Ici, il n'y a pas de lutte. L'illustrateur nous montre simplement une expédition. Les océans deviennent un champ d'exploration et d'expérimentation.

Bruguera, 1977 (fig. 15)

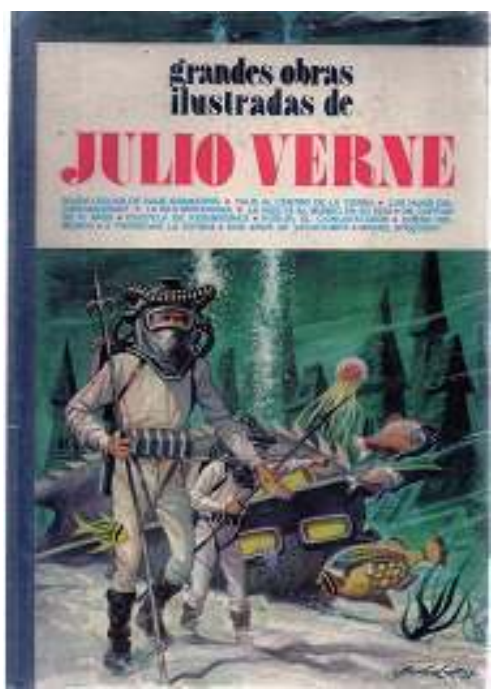

17 Cette couverture transmet l'idée de Chelebourg (1991:44): «c'est un roman de la connaissance intime de la mer ». La profondeur de la mer qui est présente tout au long du roman est ici expliquée par l'emploi de différentes tonalités vertes. Le rapport de cette 
couleur avec la sensation de sécurité provient de sa facilité de perception. Selon Max Lüscher (Aparici \& García, 1998: 90), le vert réveille la curiosité, la réflexion, l'imagination et c'est ce que l'illustrateur de cette couverture a réussi à transmettre. Il nous offre une aventure sous les mers.

Cette idée se répète sur la couverture de $1980_{b}$, publiée par la maison d'édition Roma, dans la collection « Album juvenil Roma » (fig. 16).

Roma, $1980_{b}$ (fig. 16)

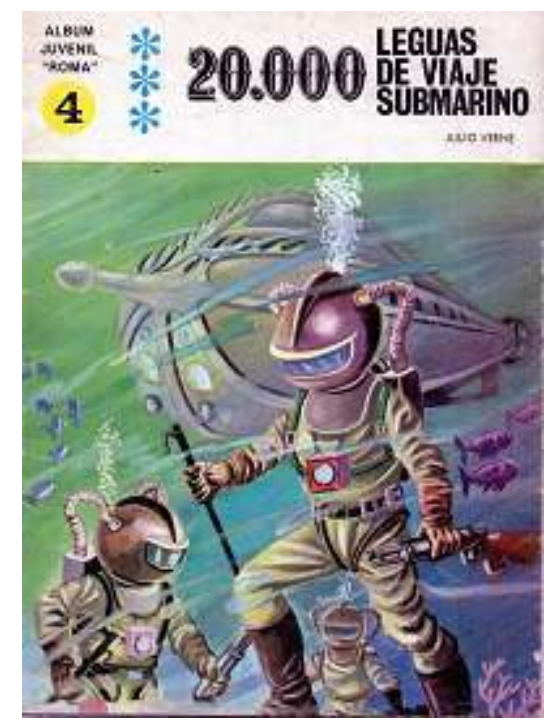

Soriano en est l'adaptateur et l'illustrateur. La similitude entre ces deux dernières couvertures est évidente et on peut supposer que Soriano a eu, peut-être, la même idée que Bernal.

La couverture de 1977 (fig. 17) de l'illustrateur Bernal a des rapports bien clairs que celle qu'il avait créée en 1970, aussi chez Bruguera, pour la collection Joyas literarias juveniles. Cependant, nous pouvons y voir une grande différence: si le Nautilus n'est pas représenté, par contre, il y a un cimetière marin. Or, comme le signale Clément (p.58), « la terre, au fond de la mer, où l'on enterre les morts : voilà la véritable profondeur ». Disons que cette couverture transmet d'une façon aimable la scène racontée par Jules Verne (1871, pp. 198-199). Voici l'illustration de Neuville chez Hetzel (fig. 18)

$\mathrm{Au}$ milieu de la clairière, sur un piédestal de rocs grossièrement entassés, se dressait une croix de corail, qui étendait ses longs bras qu'on eût dit faits d'un sang pétrifié.

Sur un signe du capitaine Nemo, un de ses hommes s'avança, et à quelques pieds de la croix, il commença à creuser un trou avec une pioche qu'il détacha de sa ceinture.

Je compris tout! Cette clairière c'était un cimetière, ce trou, une tombe, cet objet oblong, le corps de l'homme mort dans la nuit! Le capitaine Nemo et les siens venaient enterrer leur compagnon dans cette demeure commune, au fond de cet inaccessible Océan! 
Bruguera, 1977 (fig. 17) -

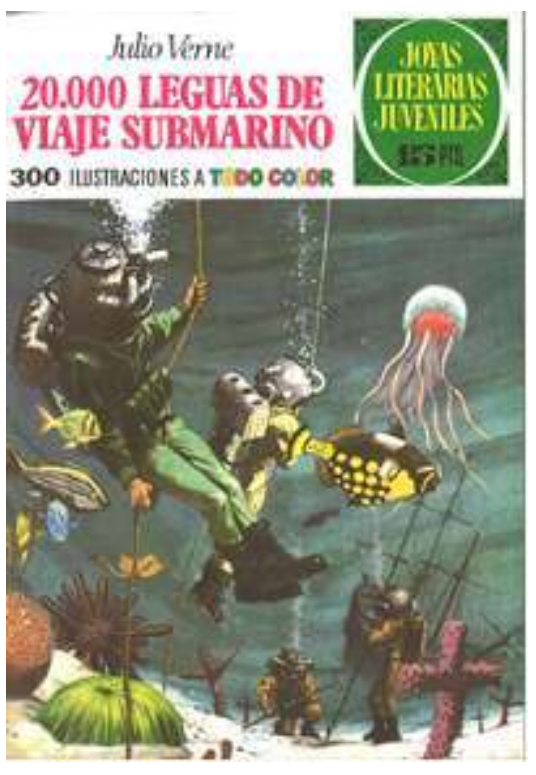

- Hetzel, 1871 (fig. 18)

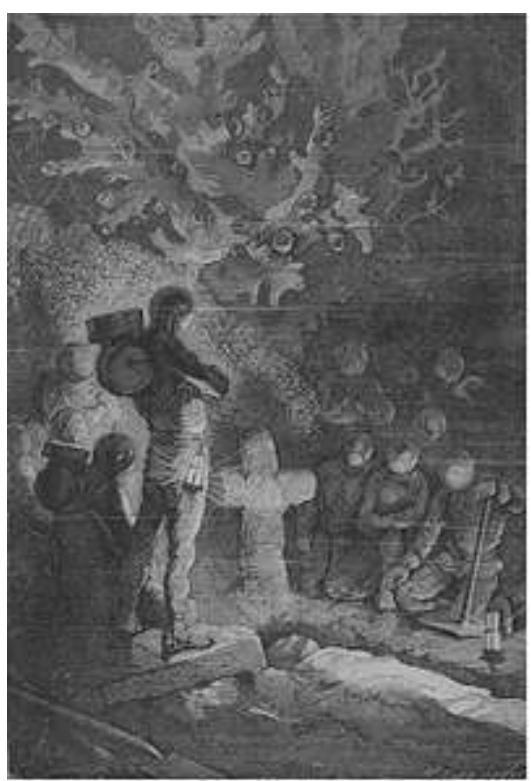

Le journal « el Periódico », en 1993, publia cette même adaptation, en fascicules (numéro $16 \mathrm{du}$ premier livre) tout en gardant la couverture (fig. 19). 


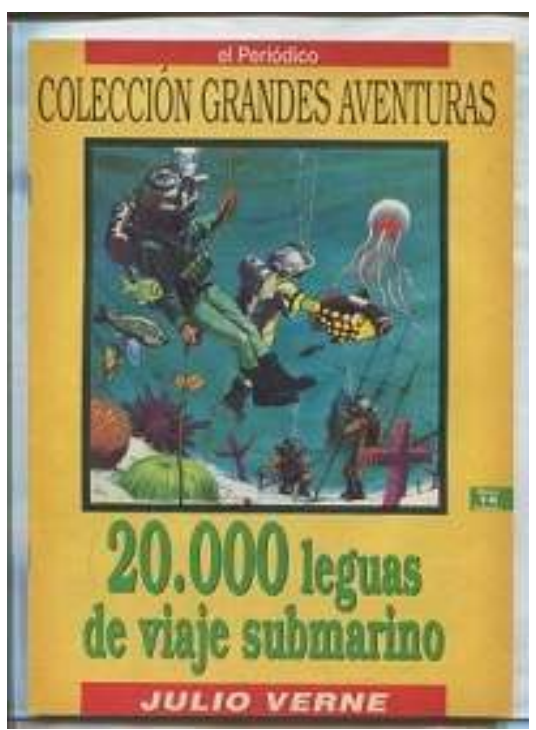

La couverture suivante de $1980_{\mathrm{a}}$ a été créée par Pedro Ruiz pour la maison d'édition Bruguera (fig. 20). Heliodoro Lillo en est l'adaptateur. Tout au long du texte plusieurs animaux sont nommés : araignée de mer, requins, oiseau de Paradis, sanglier, cachalot, baleines, lamantin, poulpe, mais l'illustrateur a choisi comme motif central une loutre de mer. Et ce n'est pas l'animal le plus important du récit. Cependant, Jules Verne le nomme six fois et en fait une belle description d'une douzaine de lignes (1871:131).

Bruguera, 1980 (fig. 20)

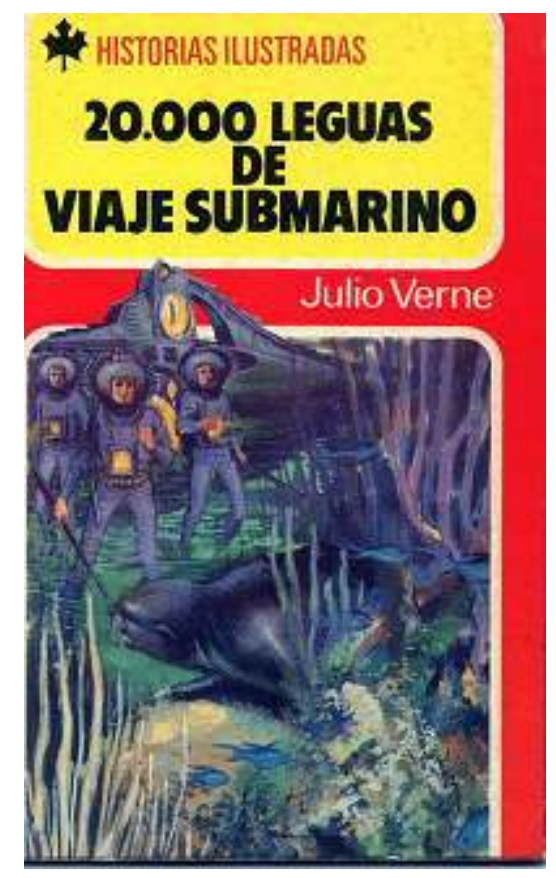

L'illustrateur fait une distribution de la couverture assez étonnante: avec un encadrement rouge, elle nous attire vers le titre en noir, couleur qui représente l'obscurité absolue, presque violente. Pedro Ruiz utilise différentes tonalités de la même couleur pour présenter le Nautilus, les plongeurs, la loutre et la mer. 
Mais s'il y a une couverture qui n'exprime pas ce que Jules Verne avait décrit dans Vingt mille lieues sous les mers : c'est celle de l'album de vignettes de la collection « chocolates Lloveras / chocolat LLoveras» (fig. 21). On peut observer des plongeurs dans un contexte industriel marin et ils s'y intéressent uniquement à la récupération des trésors sousmarins.

Chocolates Lloveras, 1965 ? (fig. 21)

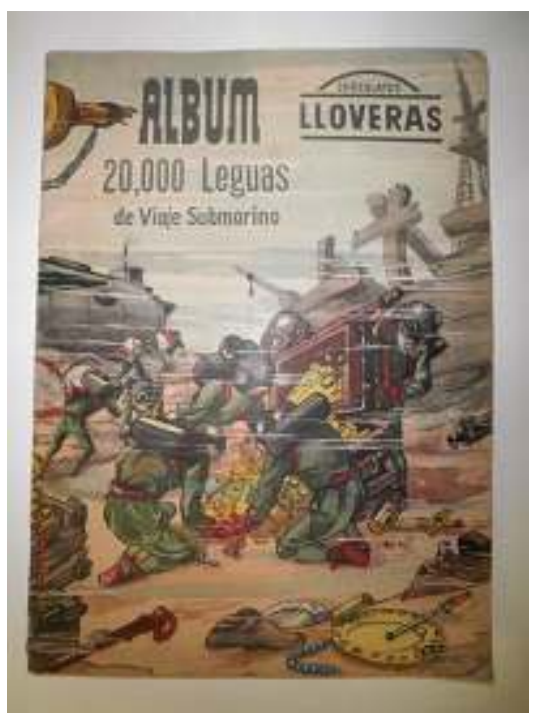

Nous pouvons conclure que les couvertures sont la porte d'entrée de l'adaptation. L'image d'une couverture est une unité de représentation qui remplace la réalité du récit, mais c'est ainsi qui nous parvenons à imaginer ce qui se trouve derrière elle. Par ailleurs, nous devons accepter l'idée qu'une représentation visuelle, pour nous les couvertures, est une structure qui transmet une certaine connaissance faite par une personne pour une raison concrète. Mais cette image, généralement, ne coïncide pas avec celle que nous aurions faite.

À travers l'analyse de toutes ces couvertures d'adaptations de Vingt mille lieues sous les mers, nous avons observé que les illustrateurs considèrent les poulpes et les plongeurs les deux motifs les plus représentatifs de ce roman. Nous avons également constaté que la représentation visuelle est presque toujours aimable. Elle ne veut pas exprimer trop de violence et l'emploi des différentes couleurs y contribue. En tout cas, le graphisme permet de nuancer une vision moins sympathique ou plus désagréable de ces couvertures.

Finalement nous pouvons affirmer que l'imagination de l'illustrateur se confrontera toujours avec celle du lecteur. Avec le choix de certains motifs pour la couverture, l'illustrateur peut modifier le sens de l'histoire écrite : le lecteur pense qu'il va trouver une aventure sous-marine où les poulpes ont un rôle principal et que les plongeurs vont subir des expériences extraordinaires. Il peut avoir des surprises. En tout cas, il aura bien d'autres lectures possibles. Pour aboutir au même résultat, il y a trois imaginations qui avancent par des sentiers bien différents mais qui s'entrecroisent: la première, imagination de l'écrivain, Jules Verne, la deuxième celle de l'illustrateur des couvertures et une troisième, celle du lecteur. En analysant ces couvertures, ces trois imaginations se heurtent-elles involontairement ou délibérément? 


\section{BIBLIOGRAPHIE}

ACASO, María (2009). El Lenguaje visual. Barcelona : Ediciones Paidós Ibérica.

APARICI, Roberto \& GARCÍA MATILLA, Agustín (1998). Lectura de imágenes. 3ª ed. Madrid : Ediciones de la Torre.

BARTHES, Roland (1964). «Rhétorique de l'image », Communications, no 15, pp. 40-51.

BAUdelaire, Charles (1976) Euvres Complètes, T. II. Paris: Gallimard, Bibliothèque de la Pléiade.

CHELEBourG, Christian (1991). «Vingt mille lieues sur un canapé », Bulletin de la Société Jules Verne, no 98, pp. 35-47.

CLÉMENT, Stéphanie (2013). « La double profondeur dans Vingt mille lieues sous les mers ou le sens des limites », Verniana, vol.6, pp. 55-64. [disponible le 30 juin 2018] <URL : http:// www.verniana.org/volumes/06/A4/Clement.pdf>.

COMPÈRE, Daniel (2005). « À travers les océans ", in Australian Journal of French Studies, no 42-3, pp. 284-294. < https://doi.org/10.3828/AJFS.42.3.284>.

FRESNAUlT-DeRUelle, Pierre (1970). « Le verbal dans les bandes dessinées », Communications, nº 15, pp. $145-161$.

FRESNAUlt-DeRUelle, Pierre (2009). La Bande dessinée. Paris : Armand Colin.

L. F. CAO, Marián (1998). « La Retórica visual como análisis posible en la didáctica del arte y de la imagen ", Arte, Individuo y Sociedad, nำ10. Servicio de Publicaciones. Universidad Complutense, pp. 39-62. [disponible le 30 juin 2018] <URL : http://revistas.ucm.es/index.php/ARIS/article/view/ ARIS9898110039A/5963>.

O'CONNOR, Robert (2009). « Nemo, the Nautilus and the Triumph of the Instru-mented Will », Verniana, vol. 2, pp. 125-132. [disponible le 30 juin 2018] <URL : http://www.verniana.org/ volumes/02/A4/Oconnor.pdf>.

VERNE, Jules (1871). Vingt mille lieues sous les mers. Paris : J. Hetzel.

\section{Bibliographie / Adaptations de 20.000 lieues sous les mers}

20.000 leguas de Viaje Submarino. Album (1965?). Barcelona: Chocolates Lloveras.

VERNE, Julio (1946). « Vinte mil leguas submarinas ». Diabrete, Lisboa: E.N.P.

VERNE, Julio (1955 ). 20.000 leguas de viaje submarino. Barcelona : Editorial Valor. Collection Aventuras fantásticas, 8 fascicules. Adaptation Lasting Grumbler, illustrations Francisco Javier.

VERNE, Julio $\left(1955_{\mathrm{b}}\right)$. Veinte mil leguas de viaje submarino. Barcelona : Editorial Bruguera. Collection Historias. Traduction Heliodoro Lillo Lutteroth, illustrations Julio Vivas García.

VERNE, Julio (1959 ${ }_{\mathrm{a}}$ ). Veinte mil leguas de viaje submarino. Barcelona : Editorial Bruguera. Collection Iris, $\mathrm{n}$ 23. Adaptation Heliodoro Lillo Lutteroth, illustrations intérieures et de la jaquette T. Marco et V. Roso.

VERNE, Julio (1959 $)$. 20.000 leguas de viaje submarino. Valencia : Editorial Valenciana. Adaptation Carlos de Monterroble, illustrations Brocal Remohi. 
VERNE, Julio (1967). Veinte mil leguas de viaje submarino. Barcelona : Editorial Bruguera. Collection Historias Selección, Serie Julio Verne, nำ1. Adaptation Heliodoro Lillo Lutteroth, illustrations intérieures Julio Vivas García, illustration de la jaquette E. Ferrán.

VERNE, Julio (1970). Veinte mil leguas de viaje submarino. Barcelona : Editorial Bruguera. Collection Joyas literarias juveniles, Serie Julio Verne, $\mathrm{n}$ ำ. Adaptation José Antonio Vidal Sales, illustrations intérieures Vicente Torregrosa Manrique, illustrations $1^{\text {ère }}$ de couverture Antonio Bernal Romero. VERNE, Julio (1972). Veinte mil leguas de viaje submarino. Barcelona : Editorial Bruguera. Collection Historias Selección, Serie Julio Verne, nำ. Adaptation Heliodoro Lillo Lutteroth, illustrations intérieures Julio Vivas García, illustrations $1^{\text {ère }}$ de couverture Tomás Porto.

VERNE, Julio (1971). 20.000 leguas de viaje submarino. Madrid : Ediciones Susaeta.

Collection Historietas, serie naranja, Adaptation et illustrations Coiras.

VERNE, Julio (1976). 20.000 leguas de viaje submarino. Bilbao : Ediciones Laida. Collection Ediclas, №16. Adaptation Beaumont, illustrations Julio Bosch.

VERNE, Julio (1977). 20.000 leguas de viaje submarino. Barcelona : Editorial Bruguera. Collection Grandes obras ilustradas de Julio Verne. Adaptation José Antonio Vidal Sales, illustrations intérieures Vicente Torregrosa Manrique, illustrations $1^{\text {ère }}$ de couverture Antonio Bernal. VERNE, Julio (1980a). 20.000 leguas de viaje submarino. Barcelona : Editorial Bruguera. Collection Historias ilustradas nำ1. Adaptation Heliodoro Lillo, illustrations intérieures Julio Vivas, illustrations $1^{\text {ère }}$ de couverture Pedro Ruiz.

VERNE, Julio (1980b). 20.000 leguas de viaje submarino. Barcelona : Editorial Roma. Collection Album Juvenil « Roma » nํㅜ. Adaptation Soriano, illustrations D. Soriano.

VERNE, Jules (1982). 20.000 léguas submarinas. Bilbao : Editorial Edinter. Collection Juvenil Laida, n7. Adaptation de Heliodoro Lillo Lutteroth, illustrations de Julio Vivas García.

VERNE, Julio (1983). 20.000 leguas de viaje submarino. Barcelona : Editorial Bruguera. Collection Historias Infantil. Adaptation de José Manuel Gómez Fábregas, illustrations de Julio Vivas, illustrations $1^{\text {ère }}$ de couverture Antonio Bernal.

VERNE, Julio (1993). 20.000 leguas de viaje submarino. Barcelona : Ed Zeta/El Periodico.

\section{RÉSUMÉS}

La première de couverture de l'œuvre de Jules Verne appelle aussitôt à notre imagination puisqu'elle nous permettra de construire l'histoire que nous allons découvrir à l'intérieur du livre. Nous analyserons l'image initiale de Vingt mille lieues sous les mers pour voir si les premières de couverture « imaginées " par les illustrateurs ont cette fonction - de découverte, de voyage et de (re)construction imaginaire - et si l'intuition du lecteur, en les regardant d'emblée, coïncide ou pas - avec ce que l'auteur et l'adaptateur ont voulu transmettre.

The front cover help developing our imagination to build the story we are going to discover. We analyze them to see if these covers "imagined" by the illustrators really have this function and if the reader's intuition, when looking at them, coincides with what the author, Jules Verne, and later the adapter wanted to transmit. 
INDEX

Mots-clés : Verne (Jules), adaptations, imagination, première de couverture

Keywords : Verne (Jules), adaptation, imagination, front cover

\section{AUTEURS}

MARÍA-PÍLAR TRESACO

Université de Saragosse

ptresaco[at]unizar.es

\section{ANA-ISABEL MONIZ}

Université de Madère

anamoniz[at]uma.pt

T3AxEL 